\title{
L'Abolition de 1848 dans la littérature des Antilles françaises
}

\author{
Chantal Claverie \\ Université des Antilles et de la Guyane
}

La littérature, dans son sens le plus général, peut être envisagée tour à tour comme un témoignage, un document, qui rend compte du vécu socio-historique et comme un absolu poétique, un monument, qui sert de référence dans la mesure où l'écriture se dégage de la réalité circonstancielle. En ce qui concerne l'abolition de 1848, ces deux aspects se retrouvent à des degrés variables dans la production littéraire des Antilles. Celle-ci est en effet historiquement liée aux deux abolitions de l'esclavage: la première, proclamée par la Convention le 4 février 1794 mais dont les décrets resteront sans effets, et la seconde, celle de 1848, oeuvre de la Seconde république, imposée grâce aux efforts conjugués de quelques humanistes métropolitains dont la figure la plus représentative est sans conteste celle de Victor Schoelcher; grâce aussi aux masses serviles des colonies avec le soutien de quelques mulâtres et - mais ils sont très peu nombreux - de colons blancs libéraux.

La production littéraire spécifiquement antillaise commence en effet en 1806, avec le roman de Prévost de Sansac, Comte de Traversay, Les Amours de Zémédare et Carina et jusqu'en 1848 , elle sera à la seule initiative des créoles blancs, véhiculant à loisir les préjugés racistes et les stéréotypes les plus dévalorisants sur les esclaves nègres et sur les hommes de couleur libres, prenant bien soin de les classifier en sous-catégories à partir de critères coloristes. Je fais ici allusion au délire classificatoire de Moreau de Méry, un colon blanc qui imposa aux colonies 13 dénominations pour désigner les sang-mêlé. Après 1848 , les conditions de l'énonciation littéraire sont sensiblement différentes. La crise du discours du colon et l'émergence progressive d'une littérature assumée par les hommes de couleur sont des facteurs significatifs dans la perception même de l'émancipation.

C'est pourquoi j'aborderai successivement d'une part, l'abolition comme facteur de la production littéraire puis d'autre part, l'abolition comme thème de cette production. 


\section{1) L'Abolition comme facteur de la production littéraire}

\section{a) Les signes avant-coureurs de l'abolition}

Dans les années 1830, alors que se profile l'abolition de l'esclavage - abolition qui se met déjà en place dans les colonies anglaises dès 1833 - la littérature antillaise envisage les mutations décisives que vont connaître les Antilles françaises. Au delà de la libération des esclaves se pose déjà, de manière plus ou moins explicite la question de l'assimilation des Colonies à l'Europe. Les écrivains prennent conscience que les îles, perdant leur spécificité, sont en train de s'aligner sur les valeurs et les normes européennes.

C'est ainsi qu'en 1835, deux auteurs, des créoles blancs, apportent deux éclairages différents sur cette période historique. D'une part, dans un roman intitulé Les Créoles ou la vie aux Antilles, Joseph Levilloux, prend le parti de l'évolution historique qui depuis la révolution de 1789 condamne l'ancien système esclavagiste fondé sur la discrimination raciale. Toutefois, cet auteur ne s'engage pas dans le mouvement abolitionniste, et, de manière significative, situe l'histoire de son roman dans les années qui ont suivi la grande révolution. La raison en est simple: il s'agit d'élaborer le mythe patriotique d'une France généreuse, octroyant liberté, égalité et fraternité, et de donner le coup d'envoi du discours de l'assimilation à une France républicaine. D'autre part Louis Maynard de Queilhe, qui, dans un ouvrage intitulé Outre-mer, trahit les crispations et les fantasmes d'une classe sociale hérissée par les discours abolitionnistes des philanthropes européens.

\section{b) Le nouveau contexte après 1848 .}

Après 1848 , la littérature antillaise reflète le débat sur les valeurs républicaines, par opposition à celles de l'Ancien Régime, lequel est associé au système esclavagiste. Deux courants se distinguent. Tout d'abord, un courant progressiste qui, même sous le Second empire, maintient cet idéal de liberté, de générosité, d'égalité raciale, de fraternité humaine, dans la lignée de l'humanitarisme des grands penseurs et écrivains romantiques comme Lamartine ou Victor Hugo. Il est possible de citer, par exemple, le créole blanc guadeloupéen libéral Octave Giraud qui en 1861 fait paraître un ouvrage, L'abolition de l'esclavage, dans lequel l'auteur cherche à réhabiliter la race noire, à combattre les clichés et les stéréotypes négatifs à leur endroit, en leur prophétisant une revanche historique. On peut encore rappeler Eugène Agricole, un nègre, premier homme de lettres de couleur, disciple de Schoelcher, admirateur de Perrinon, mulâtre et premier commissaire de la République de 1848 en l'honneur de qui il rédigea une ode "pleine de reconnaissance et d'estime," qui lutta sans cesse pour prolonger l'action de 1848 et inscrire dans les faits sociaux et dans les mentalités les principes proclamés par la Révolution. Vers la fin du XIXème siècle, on citera encore René Bonneville, blanc créole libéral martiniquais, qui épouse également la cause de la République, se 
démarquant ainsi de son milieu. Dans un roman intitulé Le triomphe d'Eglontine qui sera publié en 1899, il met en évidence l'ascension sociale des hommes de couleur dans le cadre de la Troisième République (1870-1940

Ensuite, le courant des nostalgiques de l'Ancien Régime qui, sans bien sûr remettre en cause directement l'abolition de l'esclavage, marquent toutefois les effets néfastes de l'égalité civique qui a été proclamée ou expriment leur suspicion envers l'aptitude des nègres à prendre en main leur destin. Ce point de vue est celui du créole blanc martiniquais E. Lemerle qui écrit en 1865 Les loisirs $d^{\prime} u n$ aveugle, recueil poétique dans lequel il développe ses réticences quant à la liberté octroyée à des noirs dont il doute des capacités à s'intégrer à une société civilisée et organisée selon le principe de la Raison, selon le stéréotype bien connu que les nègres occupent exclusivement la sphère de l'instinct de l'immaturité propre à une sous-humanité . Quant à Rosemond de Beauvallon, romancier blanc créole guadeloupéen, qui écrit en 1885 Hier! Aujourd'hui! Demain! ou les Agonies créoles, il déplore l'intrusion des valeurs et des forces métropolitaines qui désorganisent et altèrent l'ancienne société créole. Même un écrivain et journaliste aussi détaché des Antilles françaises dont il est pourtant originaire, Louis Xavier Eyma, ne peut s'empêcher d'envisager les conséquences, négatives par bien des aspects, de l'abolition de l'esclavage dans une chronique de la vie aux îles Les Peaux noires en 1857. Son détachement masque mal un sentiment de déclassement historique.

\section{c) Les transpositions littéraires vers 1900}

A la fin du XIXème siècle, la littérature transpose au plan esthétique la problématique sociohistorique initiée par 1848. L'écriture exotique et parnassienne d'auteurs comme Daniel Thaly ou Victor Duquesnay suggère le désir de s'abstraire du grand mouvement d'émancipation qui a été mis en branle par l'abolition. Tout en proposant une représentation purement plastique du monde antillais, ces auteurs proposent plus ou moins implicitement la fiction d'une société pluri-ethnique harmonieuse, laissant dans l'ombre la montée en puissance du prolétariat noir.

\section{2) L'Abolition comme thème littéraire.}

En focalisant son propos sur le 27 avril 1848, la littérature semble adopter une double perspective: celle de la tradition schoelchérienne, pour reprendre une expression du chercheur Jack Corzani, et d'autre part celle de la réinterprétation antillaise à partir d'un point de vue plus spécifiquement insulaire.

\section{a) La tradition schoelchérienne}

Elle met l'accent sur la tradition humaniste de la France des Droits de l'homme, incarnée aux yeux des Antillais par le combat inlassable de Victor Schoelcher en faveur de l'émancipation 
des esclaves. La traduction littéraire de cet idéal se marque notamment dans la production romanesque du XXème siècle par l'évocation des soulèvements serviles de mai 1848, courtcircuitant et actualisant le décret du 27 avril 1848. Au plan du récit, l'Abolition se révèle comme un dénouement heureux d'une période de luttes et de tensions exacerbées entre les colons et les esclaves. Le premier auteur à utiliser ce procédé est Drasta Houël, écrivain békée martiniquaise qui présente en 1925 un roman intitulé Cruautés et tendresses qui se clôt sur l'évocation de l'abolition, point final de la révolte des nègres et point de départ de leur lutte pour l'affirmation de leur identité et de leurs traditions. Léonard Sainville, dans le roman Dominique, Nègre esclave, paru en 1951, met plutôt l'accent sur le fait que l'Abolition fut arrachée par les esclaves, conquise donc tout autant qu'elle avait été accordée par la métropole. Le héros, Dominique, après s'être évadé de la prison de Fort-Royal, participe aux émeutes et se retrouve le 23 mai sur la place Bertin au milieu d'une foule déchaînée : "libre, farouche, il applaudit la révolution victorieuse et la liberté proclamée...". Mais l'Abolition apparaît bel et bien comme le terme naturel de la révolte nègre et pour ainsi dire l'épilogue obligé du roman du marronnage. Dans la même veine, César Pulvar, en 1957 fait paraître un roman $D^{\prime} J h e ́ b o$, le Léviathan noir. Là encore, le héros est un marron qui après bien des combats et des aventures, découvre en 1848 la fraternité instaurée par l'Abolition:

rassemblée au son des caisses, une foule immense grouille sur la place Bertin. Noirs, békés, Mulâtres déjà fraternisent ...

La transformation du nègre marron en citoyen français apparaît comme l'un des mythes littéraires antillais qui exprime le mieux le paradoxe d'une assimilation-revendication et d'une subjugation masquée par les idéaux de Liberté et d'Egalité.

\section{b) la réinterprétation antillaise de l'histoire}

D'autres écrivains antillais, tout en reconnaissant l'apport de Schoelcher et l'idéal humaniste de la France des Droits de l'homme et du citoyen, soulignent la spécificité de l'histoire antillaise, même si celle-ci est en relation étroite avec les événements de la métropole. Significatif est donc le discours prononcé à la Sorbonne, le 27 avril 1948 par Aimé Césaire, sous le titre Commémoration du centenaire de l'abolition de l'esclavage. Césaire affirme d'abord que l'abolition constitue l'essentiel de l'oeuvre de la Seconde République (1848-1851):

Je dis que c'est la gloire de la révolution de 1848, la gloire du gouvernement provisoire issu du soulèvement de février, de n'avoir pas supporté que ce crime contre l'homme, toléré et encouragé par la monarchie, déshonorât la République et dénaturât plus longtemps la civilisation . ${ }^{1}$ 
L'écrivain se réfêre ensuite, après avoir souligné l'ambivalence de ce XIXème siècle, à la fois progressiste et colonialiste, au peuple de Paris, aux ouvriers qui, par une pétition en 1844 réclamèrent l'abolition de l'esclavage: "C'est pour obéir au grand principe de la fraternité humaine, que nous venons vous faire entendre notre voix en faveur de nos malheureux frères, les esclaves." (Césaire 409) Ensuite Césaire fait l'éloge de Schoelcher qui représente la conscience de cette société, Schoelcher prenant la défense de "l'homme indigène, la victime de ce heurt de deux mondes que constitue la colonisation." Le chantre de la Négritude met enfin en évidence le fait que l'abolition de 1848 constitue pour les Antillais une sorte de pacte républicain:

Emancipation non pas concédée, d'en haut, non pas octroyée dédaigneusement, mais émancipation républicaine, émancipation révolutionnaire, sans "apprentissage" à la manière anglaise, sans "éducation préalable," sans "travail forcé," "sans limitation de droits." (Césaire 413)

L'essentiel de ce discours est pourtant ailleurs car il invite les Antillais, les Guyanais et les Réunionnais, et donc les écrivains issus de ces territoires, à s'émanciper de la discursivité historique de l'Europe pour devenir leur propre sujet:

Et ils savent désormais qu'aucun destin ne pèse sur eux, qu'ils sont les maîtres de leur histoire pour le mal comme pour le bien.

Et quand ils jettent un regard en arrière, ils ne sont pas tentés d'être ingrats, mais à la lumière même de ce passé, ils apprennent à considérer que la vraie émancipation n'est pas celle qui se décrète, mais celle que l'homme conquiert sur lui-même, qu'elle n'est pas derrière eux, et que c'est à eux qu'il appartient de la préparer en communion avec le peuple de France, dans le sillage lumineux de 1848. (Césaire 415)

Ce message n'échappe pas à Glissant, lorsqu'il rédige Le quatrième siècle, qui paraîtra en 1964 et qui est une chronique qui retrace du point de vue des Antillais leur histoire depuis les premiers temps de l'Etablissement aux îles. Le chapitre $\mathrm{X}$ de ce roman évoque le travail de deux commis de l'Etat-civil, chargés après l'abolition de 1848, d'enregistrer les noms des anciens esclaves - nouveaux citoyens. Ces deux fonctionnaires semblent détenir le pouvoir de nommer et, par là même de dicter leur destin aux nègres, mais ils subissent finalement l'ascendant de la foule à laquelle se sont mêlés les anciens marrons:

Les anciens esclaves des Plantations étaient là, y compris les femmes. Mais aussi, majestueux dans leur haillons, traînant comme une parure de dignité leur 
vie et leur dénuement, et les seuls d'ailleurs à être armés de coutelas, les marrons. Dans le contexte de loques et de hardes, ils trouvaient le moyen d'être à la fois les plus démunis et les plus superbes. Ils s'en venaient par petits groupes comme autant d'îles fermes dans la mer bouillonnante. Ils ne parlaient pas, ne gesticulaient pas, et on pouvait respirer dans leur sillage comme un relent de crainte, vite balayée par l'excitation de la journée. Les marrons étaient partagés entre la satisfaction de celui qui voit légitimer son existence ou ratifier son passé, la curiosité d'aller-et-venir sans souci dans la dédale des ruelles qu'ils avaient naguère parcourues à la dérobée, et la vague regret des jours révolus, quand le danger de vivre les élisait au plus haut de l'ordre de vie. $^{2}$

Le vieux planteur La Roche va mourir au même moment sur un navire négrier qu'il a affrété pour une ultime traite de contrebande. Cette mort est symbolique d'une mutation décisive. Les nègres qui sont devenus des citoyens ont désormais un nom officiellement reconnu:

Celui qui porte un nom est comme celui qui apprend à lire: s'il n'oublie pas le nom, l'histoire réelle du nom, et s'il ne désapprend pas de lire, il se hausse. Il se met à connaître une mère, un père, des enfants : il apprend à vouloir les défendre. Il quitte le trou béant des jours et des nuits, il entre dans le temps qui lui réfléchit un passé, le force vers un futur . (Glissant 180)

Le Guadeloupéen Daniel Maximin, dans une perspective un peu différente, écrit également la chronique des Antilles dans L'Isolé Soleil en 1981. Il met l'accent, comme l'avait fait avant lui Léonard Sainville sur l'appropriation de la liberté par les noirs eux-mêmes, par anticipation, avant même l'arrivée des commissaires de la République qui ne peuvent que dire:

Je croyais descendre sur une terre d'esclaves, et je mets le pied sur une terre de liberté. De tous les faits accomplis, il n'en est pas plus acceptable, assurément que cette anticipation de la délivrance de mes frères noirs. Homme et abolitionniste, je m'en réjouis. Salut donc à la liberté irrévocablement acquise aujourd'hui par la double consécration du fait et du droit. C'est par le travail, frères noirs, que se manifestera votre reconnaissance envers vos vrais amis, envers vos libérateurs. Par le travail et par l'ordre vous conserverez à la France ses colonies. ${ }^{3}$

Mais l'originalité de Maximin réside dans la mise en perspective de la première émancipation de 1794 et de la seconde de 1848. La figure de Schoelcher est donc symboliquement rattachée à celle de Delgrès qui, en 1802, avait conclu son épique résistance par la tragédie de Matouba. L'auteur guadeloupéen laisse ainsi entendre que les abolitionnistes de 1848 viennent parachever 
l'hérö̈que combat d'Ignace et de Delgrès. Il suggère aussi donc aussi que les Antillais doivent s'approprier et domicilier aux Antilles les valeurs les plus hautes y compris contre ceux qui les ont initiés comme ces insurgés de 1802, luttant, au chant de la Marseillaise, contre Richepanse, pacificateur envoyé par la métropole. L'auteur rappelle le célèbre discours placardé dans les rues de Basse-Terre qui commençait par ces mots:

C'est dans les plus beaux jours d'un siècle à jamais célèbre par le triomphe des lumières et de la philosophie qu'une classe d'infortunés qu'on veut anéantir se voit obligée d'élever sa voix vers la postérité, pour lui faire connaître lorsqu'elle aura disparu, son innocence et ses malheurs. (Maximin 48)

Le Martiniquais Vincent Placoly publie en 1983 un roman qui tient à la fois de la chronique et du journal : Frères Volcans dont le sous-titre est chronique de l'abolition de l'esclavage. L'originalité de ce texte vient d'abord de ce qu'il se présente comme le journal d'un béké qui transcrit sa vision des événements de 1848 alors même que l'auteur est un antillais de couleur. Le ton adopté, qui est celui de la distanciation critique, serait le fait de la maladie du narrateur et de son retrait de la vie économique de la plantation, mais en réalité, les réflexions du personnage béké, aussi bien d'ordre socio-politique que d'ordre éthique, s'enrichissent des analyses de l'auteur. Ainsi les voix de Placoly et du personnage central, qui reste étrangement anonyme, se mêlent intimement pour susciter une prise de conscience de l'affirmation historique de la personnalité antillaise:

Pour que les générations futures comprennent les événements de Mai qui ont si profondément secoué la colonie, il faut se dire que les propriétaires n'ont jamais eu peur de leurs esclaves. Le maître considère la servilité comme naturelle. L'exemple de leur père les a formé à l'autorité du despote, qui ne se dément pas. Ils ont coutume de dire : "On ne transporte pas des peuples courageux à fond de cale pour les vendre comme des marchandises, avec de la morue salée et du pétun. Les Indiens ont failli désespéré notre race parce qu'ils parlaient le langage des serpents, et qu'ils savaient pousser le combat d'homme à homme jusqu'à la dent déchirante des récifs."

Et les Pierrot? Et les Michel ?Et les autres Makendal ? Et ceux dont les noms ne sont pas connus? Ils savent que ceux-là regardent avec des yeux d'autour la richesse brûlable de leurs propriétés. Lorsqu'on leur pose cette question ils répondent ceci: "Certes, un exemple sur des millions fait chaque individu côtoyer une fois l'énergie qui le pousse à se délivrer, soi et les autres. Mais c'est un exemple sur des millions. D'ailleurs, quand l'esclave se lève les armes jusqu'au bout contre son maître, je dis bien jusqu'au bout, chacun gagne en combattant à mort un peu de cette humanité qui fait que tous les hommes sont 
frères."

Vincent Placoly, refusant de se laisser enfermer dans la dialectique hégélienne du maître et de l'esclave, aborde la question de la liberté d'un autre point de vue: un seul esclave marron suffit à éveiller, dans la masse servile, l'aspiration à l'émancipation. Le romancier souligne donc que c'est moins la situation matérielle de l'esclave qui est en cause que son statut qui entérine un déficit ontologique: "si l'esclavage renferme toutes les misères du prolétariat, l'esclave souffre d'un surplus de misère, car son existence est mêlée de mal." (Placoly 60) La leçon du 27 avril et des événements de mai 1848 n'est pas seulement celle que l'on peut consigner dans les écrits, elle est cette expérience existentielle qui se transmet à travers la sensibilité collective et ce que l'on pourrait appeler le reliquaire des corps:

Si les esclaves n'ont rien laissé d'écrit, comment retrouver le chemin de leur civilisation sinon en écoutant, pour le décrypter, le code de tout ce qui peut rester d'immortel en nous, d'inaltérable, le legs de tous ces corps qui découvraient, du fond de leur caverne, l'idée de la liberté ? (Placoly 124)

L'Abolition a donc rassemblé symboliquement les différentes ethno-classes antillaises mais surtout les différentes composantes de la communauté de couleur. : l'esclave ou nègre d'habitation, le marron qui vit aux franges de la Plantation, les libres de couleurs qui peuvent être considérés comme des archétypes du colonisé (citoyens de seconde zone). Le risque était donc d'aligner le destin de tous les nègres au niveau de ce dernier, c'est-à-dire à celui d'homme de couleur assimilé, aliéné, dépersonnalisé. Or c'est précisément en affirmant l'autonomie de son discours que ce dernier peut accéder à la liberté plénière.

Ainsi Sonny Rupaire, dont nous saluons la mémoire, considérait en 1971 que cette liberté n'était pas encore ancrée dans les consciences antillaises et qu'elle restait à naître:

O ma liberté

toi

cette enfant qui jaillira

au forceps

du bassin douloureux de la mer des Antilles

Liberté notre

$$
\hat{\mathrm{o}}^{5}
$$

Il estimait aussi que cette liberté ne serait pas octroyée par quelque héros solitaire, mais issue du peuple, ce personnage collectif, dont les trois cents martyrs réunis autour de Delgrès "les trois cents sangs giclés vers ton ciel Matouba" constituaient les prémices, comme une offrande 
sacrée pour une abolition intégrale de toute servitude.

\section{Notes}

${ }^{1}$ A. Césaire, Commémoration du centenaire de l'abolition de l'esclovage in Oeuvres complètes (Fort-de-France: Editions Désormeaux, 1976), tome 3, p.407.

${ }^{2}$ E. Glissant, Le quatrième siècle (Paris: Le Seuil, 1964), pp.176-177.

${ }^{3}$ D. Maximin, L'Isolé soleil (Paris: Le Seuil, 1981), p. 74.

${ }^{4}$ V. Placoly, Frères Volcans (Paris: La Brèche, 1983), p. 89.

${ }^{5}$ S. Rupaire, ...cette igname brisée qu'est ma terre natale (Paris: Éditions Parabole, 1971), p.55. 